\title{
DEGENERATE DISTRIBUTED CONTROL SYSTEMS WITH FRACTIONAL TIME DERIVATIVE ${ }^{1}$
}

\author{
Marina V. Plekhanova \\ Computational Mechanics Department, South Ural State University; \\ Laboratory of Quantum Topology, Mathematical Analysis Department, \\ Chelyabinsk State University, Chelyabinsk, Russia, \\ mariner79@mail.ru
}

\begin{abstract}
The existence of a unique strong solution for the Cauchy problem to semilinear nondegenerate fractional differential equation and for the generalized Showalter-Sidorov problem to semilinear fractional differential equation with degenerate operator at the Caputo derivative in Banach spaces is proved. These results are used for search of solution existence conditions for a class of optimal control problems to a system described by the degenerate semilinear fractional evolution equation. Abstract results are applied to the research of an optimal control problem solvability for the equations system of Kelvin-Voigt fractional viscoelastic fluids.
\end{abstract}

Key words: Fractional differential calculus, Caputo deivative, Mittag-Leffler function, Partial differential equation, Degenerate evolution equation, $(L, p)$-bounded operator, Optimal control, Fractional viscoelastic fluid.

\section{Introduction}

Let $\mathcal{X}, \mathcal{Y}$ be Banach spaces, $L, M: \mathcal{X} \rightarrow \mathcal{Y}$ be linear operators, ker $L \neq\{0\}, \alpha>0, m \in \mathbb{N}$, $m-1<\alpha \leq m, r \in\{0,1, \ldots, m-1\}, N:\left(t_{0}, T\right) \times \mathcal{X}^{r+1} \rightarrow \mathcal{Y}$. Denote by $D_{t}^{\alpha}$ the Caputo fractional derivative [1]. The main purpose of the paper is to study the initial value problems unique solvability to the fractional order differential equation

$$
L D_{t}^{\alpha} x(t)=M x(t)+N\left(t, x(t), x^{(1)}(t), \ldots, x^{(r)}(t)\right), \quad t \in\left(t_{0}, T\right),
$$

in the sense of the strong solutions and the solvability of optimal control problems for systems with the state that described by (0.1). Such equations are called degenerate because of degeneracy of the operator $L$ at the highest derivative. The equation with left-hand side in the form $D^{\alpha} L x$ is considered also. It has different properties beginning with the definition of a solution.

The theory of fractional differentiation in the last decades is actively used in the engineering and science problems. At first in the paper the existence of a unique solution is proved for the Cauchy problem to the nondegenerate fractional differential equation $(\mathcal{X}=\mathcal{Y}, L=I$ in (0.1)). These results are used for research of the unique solvability for the generalized Showalter-Sidorov initial value problem to the degenerate fractional differential equations. Applying the obtained statements solution existence conditions are found for a class of optimal control problems to a distributed systems described by equation (0.1) with initial conditions. Abstract results are illustrated on an optimal control problem for the equations system of Kelvin-Voigt fractional viscoelastic fluids [2].

The main condition on the operators $L, M$ in this paper is $(L, p)$-boundedness of $M$. It was introduced in [3] for the investigation of the first order degenerate equations. The conditions of the unique solution existence for the semilinear first order degenerate differential equations under this condition were studied in [4]. The solvability in the classical sense of the linear degenerate fractional equations with $(L, p)$-bounded operator $M$ was studied in the works $[5,6]$ and in [7] in the case of strongly $(L, p)$-sectorial operator. Initial boundary value problem for the linearized

\footnotetext{
${ }^{1}$ The work is supported by Laboratory of Quantum Topology of Chelyabinsk State University (Russian Federation government grant 14.Z50.31.0020).
} 
system of Kelvin-Voigt fractional fluids was investigated in [8]. The equations of form (0.1) with $(L, p)$-bounded operator $M$ and with $\alpha=m \in \mathbb{N}$ were investigated in [9]. The solvability in the sense of the classical solution for another class of degenerate fractional equations (0.1) in Banach spaces with restriction on the image of $N$ was studied in [10]. Related problems in Banach and locally convex spaces for degenerate and nondegenerate fractional order evolution equations were explored by M. Kostić [11] but for other classes of operators and using mild solution and similar notions. Note papers by A.V. Glushak $[12,13]$ devoted to some differential equations in Banach spaces with the Riemann-Liouville, Euler-Poisson-Darboux and other derivatives. In contrast to the mentioned works the results of the present paper concern the existence of a unique strong solution for semilinear degenerate evolution fractional order equations that previously were not investigated.

In the present paper, when studying optimal control problems for equations of form (0.1), we use the general scheme suggested in the monograph [14, p. 16]. It was earlier applied to optimal control problems for a degenerate distributed systems of the first order in papers [15-17]. Optimal control problems for fractional equations are poorly understood. Most of them devoted to nondegenerate equations $[18,19]$, stochastic equations [20] and others. Here a research of control problems for semilinear degenerate evolution equations that has previously not been studied is presented.

\section{Nondegenerate linear equation of fractional order}

Let $\mathcal{Z}$ be Banach space. Introduce the Lebesgue spaces $L_{q}(0, T ; \mathcal{Z})$ and for $q \in(1, \infty), k \in \mathbb{N}$ Sobolev spaces

$$
W_{q}^{k}(0, T ; \mathcal{Z})=\left\{f \in L_{q}(0, T ; \mathcal{Z}): f^{(k)} \in L_{q}(0, T ; \mathcal{Z})\right\} .
$$

Denote $g_{\delta}(t)=\Gamma(\delta)^{-1} t^{\delta-1}$,

$$
J_{t}^{\delta} h(t)=\left(g_{\delta} * h\right)(t)=\int_{0}^{t} g_{\delta}(t-s) h(s) d s, \quad \text { for } \quad \delta>0, \quad t>0 .
$$

Let $\alpha>0, m$ be the smallest positive number not exceeding $\alpha, D_{t}^{m}$ is a usual derivative of the order $m \in \mathbb{N}, J_{t}^{0}$ is the identical operator,

$$
D_{t}^{\alpha} f(t)=D_{t}^{m} J_{t}^{m-\alpha}\left(f(t)-\sum_{k=0}^{m-1} f^{(k)}(0) g_{k+1}(t)\right)
$$

is the Caputo derivative [1, p. 11].

Consider the Cauchy problem

$$
z^{(k)}(0)=z_{k}, \quad k=0,1, \ldots, m-1,
$$

for the inhomogeneous differential equation

$$
D_{t}^{\alpha} z(t)=A z(t)+f(t), \quad t \in(0, T),
$$

where $A \in \mathcal{L}(\mathcal{Z})$ (linear and bounded operator from $\mathcal{Z}$ to $\mathcal{Z}$ ), the function $f:(0, T) \rightarrow \mathcal{Z}$ is given for $T>0$.

A strong solution of the problem $(1.1)-(1.2)$ is a function $z \in C^{m-1}([0, T] ; \mathcal{Z})$, such that

$$
g_{m-\alpha} *\left(z-\sum_{k=0}^{m-1} z^{(k)}(0) g_{k+1}\right) \in W_{q}^{m}(0, T ; \mathcal{Z}),
$$


conditions (1.1) are valid and equality (1.2) holds almost everywhere on $(0, T)$.

For $\alpha, \beta>0$ denote the Mittag-Leffler function

$$
E_{\alpha, \beta}(z)=\sum_{n=0}^{\infty} \frac{z^{n}}{\Gamma(\alpha n+\beta)} .
$$

Theorem 1. Let $A \in \mathcal{L}(\mathcal{Z}), f \in L_{q}(0, T ; \mathcal{Z}), q \in(\max \{1,1 / \alpha\}, \infty)$. Then for any $z_{k} \in \mathcal{Z}$, $k=0,1, \ldots, m-1$, there exists a unique strong solution of the problem (1.1)-(1.2), it has the form

$$
z(t)=\sum_{k=0}^{m-1} t^{k} E_{\alpha, k+1}\left(A t^{\alpha}\right) z_{k}+\int_{0}^{t}(t-s)^{\alpha-1} E_{\alpha, \alpha}\left(A(t-s)^{\alpha}\right) f(s) d s .
$$

P r o o f. For $k=1,2, \ldots, m-1, l=1,2, \ldots, k$ we have

$$
\frac{d^{l}}{d t^{l}} t^{k} E_{\alpha, k+1}\left(A t^{\alpha}\right)=\sum_{n=0}^{\infty} \frac{A^{n} t^{\alpha n+k-l}}{\Gamma(\alpha n+k+1-l)}=t^{k-l} E_{\alpha, k+1-l}\left(A t^{\alpha}\right),
$$

and for $l=k+1, k+2, \ldots, m-1$

$$
\frac{d^{l}}{d t^{l}} t^{k} E_{\alpha, k+1}\left(A t^{\alpha}\right)=\sum_{n=1}^{\infty} \frac{A^{n} t^{\alpha n+k-l}}{\Gamma(\alpha n+k+1-l)}=t^{\alpha+k-l} A E_{\alpha, \alpha+k+1-l}\left(A t^{\alpha}\right) .
$$

So for $l=1,2, \ldots, m-1$

$$
\left.\frac{d^{l}}{d t^{l}} \sum_{k=0}^{m-1} t^{k} E_{\alpha, k+1}\left(A t^{\alpha}\right) z_{k}\right|_{t=0}=\left.E_{\alpha, 1}\left(A t^{\alpha}\right) z_{l}\right|_{t=0}=z_{l} .
$$

Then, using formula (1.4), we get with $l=0,1, \ldots, m-1$

$$
\left.\frac{d^{l}}{d t^{l}}\right|_{t=0} \int_{0}^{t}(t-s)^{\alpha-1} E_{\alpha, \alpha}\left(A(t-s)^{\alpha}\right) f(s) d s=0,
$$

therefore

$$
\begin{gathered}
D_{t}^{\alpha} \int_{0}^{t}(t-s)^{\alpha-1} E_{\alpha, \alpha}\left(A(t-s)^{\alpha}\right) f(s) d s= \\
=D_{t}^{m} \int_{0}^{t} \frac{s^{m-\alpha-1}}{\Gamma(m-\alpha)} d s \int_{0}^{t-s}(t-s-\sigma)^{\alpha-1} E_{\alpha, \alpha}\left(A(t-s-\sigma)^{\alpha}\right) f(\sigma) d \sigma= \\
=D_{t} \int_{0}^{t} f(\sigma) d \sigma \sum_{n=0}^{\infty} \int_{0}^{t-\sigma} \frac{A^{n}(t-s-\sigma)^{\alpha(n+1)-m} s^{m-\alpha-1}}{\Gamma(m-\alpha) \Gamma(\alpha(n+1)-m+1)} d s= \\
=D_{t} \int_{0}^{t} f(\sigma) d \sigma \sum_{n=0}^{\infty}(t-\sigma)^{\alpha n} A^{n} \int_{0}^{1} \frac{(1-\tau)^{\alpha(n+1)-m} \tau^{m-\alpha-1}}{\Gamma(m-\alpha) \Gamma(\alpha(n+1)-m+1)} d \tau= \\
=D_{t} \int_{0}^{t} f(\sigma) E_{\alpha, 1}\left(A(t-\sigma)^{\alpha}\right) d \sigma=A \int_{0}^{t}(t-s)^{\alpha-1} E_{\alpha, \alpha}\left(A(t-s)^{\alpha}\right) f(s) d s+f(t)
\end{gathered}
$$


almost everywhere on $(0, T)$.

From Hölder's inequality it follows that

$$
\begin{gathered}
\int_{0}^{T}\left\|A \int_{0}^{t}(t-s)^{\alpha-1} E_{\alpha, \alpha}\left(A(t-s)^{\alpha}\right) f(s) d s\right\|_{\mathcal{Z}}^{q} d t \leq \\
\leq\left(\frac{q-1}{\alpha q-1}\right)^{q-1} T^{\alpha q}\left(\|A\|_{\mathcal{L}(\mathcal{Z})} E_{\alpha, \alpha}\left(T^{\alpha}\|A\|_{\mathcal{L}(\mathcal{Z})}\right)\right)^{q}\|f\|_{L_{q}(0, T ; \mathcal{Z})}^{q}
\end{gathered}
$$

because $q>1 / \alpha$. Thus, function (1.3) is a strong solution of problem (1.1), (1.2).

If there exist strong solutions $y_{1}$ and $y_{2}$ of the problem (1.1)-(1.2), then their difference $z=$ $y_{1}-y_{2}$ is the solution of the Cauchy problem (1.1) with the initial data $z_{k}=0, k=0,1, \ldots, m-1$, for a homogeneous equation $D_{t}^{\alpha} z(t)=A z(t)$. Act on both sides of this equation by the operator $J_{t}^{\alpha}$ and obtain

$$
z(t)=\int_{0}^{t} \frac{(t-s)^{\alpha-1}}{\Gamma(\alpha)} A z(s) d s,
$$

because $[1$, p. 12]

$$
J_{t}^{\alpha} D_{t}^{\alpha} z=z+\sum_{k=0}^{m-1} z^{(k)}(0) g_{k+1} .
$$

By definition of a strong solution we have $z \in C([0, T] ; \mathcal{Z})$ even for $\alpha \in(0,1)$. Then

$$
\max _{t \in\left[0, t_{A}\right]}\left\|\int_{0}^{t} \frac{(t-s)^{\alpha-1}}{\Gamma(\alpha)} A z(s) d s\right\|_{\mathcal{Z}} \leq \frac{t_{A}^{\alpha}\|A\|_{\mathcal{L}(\mathcal{Z})}}{\Gamma(\alpha+1)}\|z\|_{C\left(\left[0, t_{A}\right] ; \mathcal{Z}\right)} .
$$

Therefore, the integral operator defined by the right-hand side of equality (1.5) is a contraction operator in the space $C\left(\left[0, t_{A}\right] ; \mathcal{Z}\right)$ if

$$
t_{A}<\left(\Gamma(\alpha+1) /\|A\|_{\mathcal{L}(\mathcal{Z})}\right)^{1 / \alpha} .
$$

Consequently, the unique fixed point of the integral operator is the solution $z \equiv 0$ on $\left[0, t_{A}\right]$. On the segment $\left[t_{A}, t_{2 A}\right]$ repeat the reasoning. After finite number of steps the uniqueness of the zero solution will be obtained for the homogeneous Cauchy problem on the interval $(0, T)$.

\section{The Cauchy problem for the semilinear equation}

Let $A \in \mathcal{L}(\mathcal{Z}), m \in \mathbb{N}, m-1<\alpha \leq m$. Operator $B:\left(t_{0}, T\right) \times \mathcal{Z}^{m} \rightarrow \mathcal{Z}$ be Caratheodory mapping, i.e. for all $z_{0}, z_{1}, \ldots, z_{m-1} \in \mathcal{Z}$ it sets measurable mapping on $\left(t_{0}, T\right)$ and for almost all $t \in\left(t_{0}, T\right)$ it is continuous with respect to $z_{0}, z_{1}, \ldots, z_{m-1} \in \mathcal{Z}$. Consider the Cauchy problem

$$
z^{(k)}\left(t_{0}\right)=z_{k}, \quad k=0,1, \ldots, m-1
$$

for the semilinear equation

$$
D_{t}^{\alpha} z(t)=A z(t)+B\left(t, z(t), z^{(1)}(t), \ldots, z^{(m-1)}(t)\right), \quad t \in\left(t_{0}, T\right) .
$$

A strong solution of the problem $(2.1)-(2.2)$ on the interval $\left(t_{0}, T\right)$ is a function $z \in C^{m-1}\left(\left[t_{0}, T\right] ; \mathcal{Z}\right)$, such that

$$
g_{m-\alpha} *\left(z-\sum_{k=0}^{m-1} z^{(k)}\left(t_{0}\right) g_{k+1}\right) \in W_{q}^{m}\left(t_{0}, T ; \mathcal{Z}\right),
$$


conditions (2.1) hold and almost everywhere on $\left(t_{0}, T\right)$ equality $(2.2)$ is true, $\left(\right.$ here $g_{k+1}=\left(t-t_{0}\right)^{k} / k$ !, $k=0,1, \ldots, m-1)$.

Lemma 1. Let $A \in \mathcal{L}(\mathcal{Z}), z_{0}, z_{1}, \ldots, z_{m-1} \in \mathcal{Z}, B:\left(t_{0}, T\right) \times \mathcal{Z}^{m} \rightarrow \mathcal{Z}$ be Caratheodory mapping, for all $y_{0}, y_{1}, \ldots y_{m-1} \in \mathcal{Z}$ and almost all $t \in\left(t_{0}, T\right)$ the estimate

$$
\left\|B\left(t, y_{0}, y_{1}, \ldots, y_{m-1}\right)\right\|_{\mathcal{Z}} \leq a(t)+c \sum_{k=0}^{m-1}\left\|y_{k}\right\|_{\mathcal{Z}},
$$

be satisfied, where $a \in L_{q}\left(t_{0}, T ; \mathbb{R}\right), c>0$. Then the function $z$ is a strong solution of the problem (2.1)-(2.2) if and only if $z \in C^{m-1}\left(\left[t_{0}, T\right] ; \mathcal{Z}\right)$ and on $\left[t_{0}, T\right]$ we have

$$
\begin{gathered}
z(t)=\sum_{k=0}^{m-1}\left(t-t_{0}\right)^{k} E_{\alpha, k+1}\left(A\left(t-t_{0}\right)^{\alpha}\right) z_{k}+ \\
+\int_{t_{0}}^{t}(t-s)^{\alpha-1} E_{\alpha, \alpha}\left(A(t-s)^{\alpha}\right) B\left(s, z(s), z^{(1)}(s), \ldots, z^{(m-1)}(s)\right) d s .
\end{gathered}
$$

P r o o f. Let $z$ be a solution of the problem $(2.1)-(2.2)$, then $z \in C^{m-1}\left(\left[t_{0}, T\right] ; \mathcal{Z}\right)$. In view of condition (2.3) the operator $B$ is bounded and continuous as mapping from $W_{q}^{m-1}\left(t_{0}, T ; \mathcal{Z}\right.$ ) (and also from $\left.C^{m-1}\left(\left[t_{0}, T\right] ; \mathcal{Z}\right)\right)$ to $L_{q}\left(t_{0}, T ; \mathcal{Z}\right)$. Arguing as in the proof of Theorem 1 , we find that the solution satisfies equation $(2.4)$.

Let $z \in C^{m-1}\left(\left[t_{0}, T\right] ; \mathcal{Z}\right)$ on $\left[t_{0}, T\right]$ satisfies equation $(2.4)$, then the function $B\left(\cdot, z(\cdot), \ldots, z^{(m-1)}(\cdot)\right) \in L_{q}\left(t_{0}, T ; \mathcal{Z}\right)$ and by analogy with Theorem 1 we can verify that $z$ is a strong solution of the problem (2.1)-(2.2).

The bar over a symbol will mean an ordered set of $m$ elements with indexes from 0 to $m-1$, for example, $\bar{z}=\left(z_{0}, z_{1}, \ldots, z_{m-1}\right)$. A mapping $B:\left(t_{0}, T\right) \times \mathcal{Z}^{m} \rightarrow \mathcal{Z}$ is called uniformly Lipschitz continuous in $\bar{y}$, if there exists $l>0$, such that the inequality

$$
\|B(t, \bar{y})-B(t, \bar{z})\|_{\mathcal{Z}} \leq l \sum_{k=0}^{m-1}\left\|y_{k}-z_{k}\right\|_{\mathcal{Z}}
$$

is true for almost all $t \in\left(t_{0}, T\right)$ and for all $\bar{y}, \bar{z}$ of $\mathcal{Z}^{m}$.

Theorem 2. Let $A \in \mathcal{L}(\mathcal{Z}), B:\left(t_{0}, T\right) \times \mathcal{Z}^{m} \rightarrow \mathcal{Z}$ be Caratheodory mapping, uniformly Lipschitz continuous in $\bar{y}, q \in(\max \{1,1 / \alpha\}, \infty)$, for some $\bar{v} \in \mathcal{Z}^{m} B(\cdot, \bar{v}) \in L_{q}\left(t_{0}, T ; \mathcal{Z}\right)$. Then for any $z_{0}, z_{1}, \ldots, z_{m-1} \in \mathcal{Z}$ the problem $(2.1)-(2.2)$ has a unique strong solution on $\left(t_{0}, T\right)$.

P r o o f. The uniformly Lipschitz continuity implies that for any $\bar{y} \in \mathcal{Z}^{m}$ for almost all $t \in\left(t_{0}, T\right)$ we have

$$
\|B(t, \bar{y})\|_{\mathcal{Z}} \leq\|B(t, \bar{v})\|_{\mathcal{Z}}+l \sum_{k=0}^{m-1}\left\|v_{k}\right\|_{\mathcal{Z}}+l \sum_{k=0}^{m-1}\left\|y_{k}\right\|_{\mathcal{Z}}
$$

therefore condition (2.3) is performed with

$$
a(t)=\|B(t, \bar{v})\|_{\mathcal{Z}}+l \sum_{k=0}^{m-1}\left\|v_{k}\right\|_{\mathcal{Z}}, \quad c=l .
$$


According to the statement of Lemma 1 it is sufficient to show that the equation (2.4) has a unique solution $z \in C^{m-1}\left(\left[t_{0}, T\right] ; \mathcal{Z}\right)$. In the space $C^{m-1}\left(\left[t_{0}, T\right] ; \mathcal{Z}\right)$ define an operator $F$ as

$$
\begin{gathered}
F(y)(t)=\sum_{k=0}^{m-1}\left(t-t_{0}\right)^{k} E_{\alpha, k+1}\left(A\left(t-t_{0}\right)^{\alpha}\right) z_{k}+ \\
+\int_{t_{0}}^{t}(t-s)^{\alpha-1} E_{\alpha, \alpha}\left(A(t-s)^{\alpha}\right) B\left(s, y(s), y^{(1)}(s), \ldots, y^{(m-1)}(s)\right) d s .
\end{gathered}
$$

By the proof of Theorem $1 F: C^{m-1}\left(\left[t_{0}, T\right] ; \mathcal{Z}\right) \rightarrow C^{m-1}\left(\left[t_{0}, T\right] ; \mathcal{Z}\right)$.

We denote by $F^{r}$ the $r$-th power of the operator $F, r \in \mathbb{N}$, and in further reasoning if $T-t_{0}<1$ we will replace $T-t_{0}$ by 1 . For $t \in\left[t_{0}, T\right], n=0,1, \ldots, m-1, r \in \mathbb{N}, y, z \in C^{m-1}\left(\left[t_{0}, T\right] ; \mathcal{Z}\right)$ by induction the inequality

$$
\left\|\left[F^{r}(y)\right]^{(n)}(t)-\left[F^{r}(z)\right]^{(n)}(t)\right\|_{\mathcal{Z}} \leq \frac{K^{r}\left(t-t_{0}\right)^{\alpha-m+r}\|y-z\|_{C^{m-1}\left(\left[t_{0}, T\right] ; \mathcal{Z}\right)}}{m(r-1) !}
$$

can be proved, where

$$
K=m l(\alpha-m+1)^{-1}\left(T-t_{0}\right)^{\alpha} \max _{n=0, \ldots, m-1} E_{\alpha, \alpha-n}\left(\left(T-t_{0}\right)^{\alpha}\|A\|_{\mathcal{L}(\mathcal{Z})}\right) .
$$

For $r=1, n=0,1, \ldots, m-1$ Hölder's inequality implies that

$$
\begin{gathered}
\left\|[F(y)]^{(n)}(t)-[F(z)]^{(n)}(t)\right\|_{\mathcal{Z}} \leq E_{\alpha, \alpha-n}\left(\left(t-t_{0}\right)^{\alpha}\|A\|_{\mathcal{L}(\mathcal{Z})}\right) \times \\
\times \int_{t_{0}}^{t}(t-s)^{\alpha-1-n}\left\|B\left(s, y(s), \ldots, y^{(m-1)}(s)\right)-B\left(s, z(s), \ldots, z^{(m-1)}(s)\right)\right\|_{\mathcal{Z}} d s \leq \\
\leq \frac{l\left(t-t_{0}\right)^{\alpha-m+1}\left(T-t_{0}\right)^{m-1-n}}{\alpha-m+1} E_{\alpha, \alpha-n}\left(\left(T-t_{0}\right)^{\alpha}\|A\|_{\mathcal{L}(\mathcal{Z})}\right)\|y-z\|_{C^{m-1}\left(\left[t_{0}, T\right] ; \mathcal{Z}\right)} .
\end{gathered}
$$

If for $r-1$ inequality (2.5) is valid, then

$$
\begin{gathered}
\left\|\left[F^{r}(y)\right]^{(n)}(t)-\left[F^{r}(z)\right]^{(n)}(t)\right\|_{\mathcal{Z}} \leq \frac{K}{m} \int_{t_{0}}^{t} \sum_{k=0}^{m-1}\left\|\left[F^{r-1}(y)\right]^{(k)}(s)-\left[F^{r-1}(z)\right]^{(k)}(s)\right\|_{\mathcal{Z}} d s \leq \\
\leq K \int_{t_{0}}^{t} \frac{K^{r-1}\left(s-t_{0}\right)^{\alpha-m+r-1}\|y-z\|_{C^{m-1}\left(\left[t_{0}, T\right] ; \mathcal{Z}\right)}}{m(r-2) !} d s \leq \\
\leq \frac{K^{r}\left(t-t_{0}\right)^{\alpha-m+r}\|y-z\|_{C^{m-1}\left(\left[t_{0}, T\right] ; \mathcal{Z}\right)}}{m(\alpha-m+r)(r-2) !}<\frac{K^{r}\left(t-t_{0}\right)^{\alpha-m+r}\|y-z\|_{C^{m-1}\left(\left[t_{0}, T\right] ; \mathcal{Z}\right)}}{m(r-1) !}
\end{gathered}
$$

From (2.5) it follows that for $r \in \mathbb{N}$ we have

$$
\left\|\left[F^{r}(y)\right]-\left[F^{r}(z)\right]\right\|_{C^{m-1}\left(\left[t_{0}, T\right] ; \mathcal{Z}\right)} \leq \frac{K^{r}\left(T-t_{0}\right)^{\alpha-m+r}\|y-z\|_{C^{m-1}\left(\left[t_{0}, T\right] ; \mathcal{Z}\right)}}{(r-1) !} .
$$

Therefore, if $r$ is sufficiently large, then $F^{r}$ is a strict contraction in $C^{m-1}\left(\left[t_{0}, T\right] ; \mathcal{Z}\right)$, so it has in this space a unique fixed point. It is the unique solution of the equation (2.4) in the space $C^{m-1}\left(\left[t_{0}, T\right] ; \mathcal{Z}\right)$, and, therefore, a unique strong solution of the problem (2.1)-(2.2) on the interval $\left(t_{0}, T\right)$. 
We will need solutions of the problem (2.1)-(2.2) with additional smoothness. For fractional $\alpha>1$ conditions of their existence were found in the case of incomplete equation only (without $(m-1)$-th derivative under the sign of operator $B)$.

Theorem 3. Let $\alpha>1, q>(\alpha+1-m)^{-1}, A \in \mathcal{L}(\mathcal{Z}), n \in \mathbb{N}, B \in C^{n}\left(\left[t_{0}, T\right] \times \mathcal{Z}^{m-1} ; \mathcal{Z}\right)$ be uniformly Lipschitz continuous in $z_{0}, z_{1}, \ldots, z_{m-2} \in \mathcal{Z}, f \in W_{q}^{n}\left(t_{0}, T ; \mathcal{Z}\right)$ and let for $z$ satisfying conditions (2.1) and the equation

$$
D_{t}^{\alpha} z(t)=A z(t)+B\left(t, z(t), z^{(1)}(t), \ldots, z^{(m-2)}(t)\right)+f(t)
$$

the equalities

$$
\left.D_{t}^{k}\right|_{t=t_{0}}\left[B\left(t, z(t), z^{(1)}(t), \ldots, z^{(m-2)}(t)\right)\right]=-f^{(k)}\left(t_{0}\right), \quad k=0,1, \ldots, n-1,
$$

hold. Then for every $z_{0}, z_{1}, \ldots, z_{m-1} \in Z$ there exists a unique strong solution $z$ of the problem (2.1), (2.6). Besides, $z \in C^{m-1+n}\left(\left[t_{0}, t_{1}\right] ; \mathcal{Z}\right)$.

$\mathrm{P} \mathrm{r}$ o o f. For $\alpha>1$ we have $m \geq 2$. Using equalities (2.7) and sequentially computing the derivatives of the right-hand side of (2.4), we obtain for $k \in \mathbb{N}_{0}$

$$
\begin{aligned}
& D_{t}^{m+k} \int_{t_{0}}^{t}(t-s)^{\alpha-1} E_{\alpha, \alpha}\left(A(t-s)^{\alpha}\right) B\left(s, z(s), z^{(1)}(s), \ldots, z^{(m-2)}(s)\right) d s= \\
= & \int_{t_{0}}^{t}(t-s)^{\alpha-m} E_{\alpha, \alpha-m+1}\left(A(t-s)^{\alpha}\right) D_{s}^{k+1}\left[B\left(s, z(s), \ldots, z^{(m-2)}(s)\right)+f(s)\right] d s .
\end{aligned}
$$

Remark 1. The form of the integral in the solution formula (2.4) implies the existence of singularity of a solution at $t=t_{0}$ in case of fractional $\alpha$, if conditions (2.7) isn't used.

\section{Degenerate semilinear equation}

Let an operator $L \in \mathcal{L}(\mathcal{X} ; \mathcal{Y})$ (linear and continuous from a Banach space $\mathcal{X}$ to a Banach space $\mathcal{Y}), M \in \mathcal{C l}(\mathcal{X} ; \mathcal{Y})$ (linear, closed and densely defined in $\mathcal{X}$ with image in $\mathcal{Y}$ ), $D_{M}$ is a domain of an operator $M$, endowded by the graph norm $\|\cdot\|_{D_{M}}=\|\cdot\|_{\mathcal{X}}+\|M \cdot\|_{\mathcal{Y}}$. Define $L$-resolvent set $\rho^{L}(M)=\left\{\mu \in \mathbb{C}:(\mu L-M)^{-1} \in \mathcal{L}(\mathcal{Y} ; \mathcal{X})\right\}$ of an operator $M$ and introduce the denotations $R_{\mu}^{L}(M)=(\mu L-M)^{-1} L, L_{\mu}^{L}=L(\mu L-M)^{-1}$.

An operator $M$ will be called $(L, \sigma)$-bounded, if

$$
\exists a>0 \quad \forall \mu \in \mathbb{C} \quad(|\mu|>a) \Rightarrow\left(\mu \in \rho^{L}(M)\right) .
$$

Lemma 2 [3]. Let an operator $M$ be $(L, \sigma)$-bounded, $\gamma=\{\mu \in \mathbb{C}:|\mu|=r>a\}$. Then the operators

$$
P=\frac{1}{2 \pi i} \int_{\gamma} R_{\mu}^{L}(M) d \mu \in \mathcal{L}(\mathcal{X}), \quad Q=\frac{1}{2 \pi i} \int_{\gamma} L_{\mu}^{L}(M) d \mu \in \mathcal{L}(\mathcal{Y})
$$

are projections. 
Put $\mathcal{X}^{0}=\operatorname{ker} P, \mathcal{X}^{1}=\operatorname{im} P, \mathcal{Y}^{0}=\operatorname{ker} Q, \mathcal{Y}^{1}=\operatorname{im} Q$. Denote by $L_{k}\left(M_{k}\right)$ the restriction of the operator $L(M)$ on $\mathcal{X}^{k}\left(D_{M_{k}}=D_{M} \cap \mathcal{X}^{k}\right), k=0,1$.

Theorem 4 [3]. Let an operator $M$ be $(L, \sigma)$-bounded. Then

(i) $M_{1} \in \mathcal{L}\left(\mathcal{X}^{1} ; \mathcal{Y}^{1}\right), M_{0} \in \mathcal{C l}\left(\mathcal{X}^{0} ; \mathcal{Y}^{0}\right), L_{k} \in \mathcal{L}\left(\mathcal{X}^{k} ; \mathcal{Y}^{k}\right), k=0,1 ;$

(ii) there exist operators $M_{0}^{-1} \in \mathcal{L}\left(\mathcal{Y}^{0} ; \mathcal{X}^{0}\right), L_{1}^{-1} \in \mathcal{L}\left(\mathcal{Y}^{1} ; \mathcal{X}^{1}\right)$.

Denote $\mathbb{N}_{0}=\{0\} \cup \mathbb{N}, G=M_{0}^{-1} L_{0}$. For $p \in \mathbb{N}_{0}$ the operator $M$ is called $(L, p)$-bounded, if it is $(L, \sigma)$-bounded, $G^{p} \neq \mathbb{O}, G^{p+1}=\mathbb{O}$.

For $m-1<\alpha \leq m, r \in\{0,1, \ldots, m-1\}$ consider the semilinear evolution equation

$$
D_{t}^{\alpha} L x(t)=M x(t)+N\left(t, x(t), x^{(1)}(t), \ldots, x^{(r)}(t)\right)+f(t), \quad t \in\left(t_{0}, T\right),
$$

with operators $L \in \mathcal{L}(\mathcal{X} ; \mathcal{Y})$, $\operatorname{ker} L \neq\{0\}, M \in \mathcal{C l}(\mathcal{X} ; \mathcal{Y})$, with a nonlinear operator $N:\left(t_{0}, T\right) \times$ $\mathcal{X}^{r+1} \rightarrow \mathcal{Y}$ and a function $f:\left(t_{0}, T\right) \rightarrow \mathcal{Y}$.

A strong solution of equation $(3.1)$ on the interval $\left(t_{0}, T\right)$ is a function $x \in W_{q}^{r}\left(t_{0}, T ; \mathcal{X}\right) \cap$ $L_{q}\left(t_{0}, T ; D_{M}\right), q \in(1, \infty)$, such that $L x \in C^{m-1}\left(\left[t_{0}, T\right] ; \mathcal{Y}\right)$,

$$
g_{m-\alpha} *\left(L x-\sum_{k=0}^{m-1}(L x)^{(k)}\left(t_{0}\right) g_{k+1}\right) \in W_{q}^{m}\left(t_{0}, T ; \mathcal{Y}\right),
$$

and almost everywhere on $\left(t_{0}, T\right)$ equality $(3.1)$ is true.

Let operator $M$ be $(L, \sigma)$-bounded. Consider the generalized Showalter-Sidorov problem $[21,22]$

$$
(P x)^{(k)}\left(t_{0}\right)=x_{k}, k=0,1, \ldots, m-1,
$$

for equation (3.1) on the interval $\left(t_{0}, T\right)$.

Remark 2. We have the equalities $P x=L_{1}^{-1} L_{1} P x=L_{1}^{-1} Q L x$. Therefore, the smoothness of $P x$ is not smaller than for the function $L x$.

Denote by $[\beta]$ the integer part of $\beta \in \mathbb{R}$.

Theorem 5. Let $\alpha>0, q \in(\max \{1,1 / \alpha\}, \infty), r=[(m-1) / 2]$, an operator $M$ be $(L, 0)$ bounded, an operator $N:\left[t_{0}, T\right] \times \mathcal{X}^{r+1} \rightarrow \mathcal{Y}$ be Caratheodory mapping, the equality

$$
N\left(t, z_{0}, z_{1}, \ldots, z_{r}\right)=N_{1}\left(t, P z_{0}, P z_{1}, \ldots, P z_{r}\right)
$$

with some $N_{1}:\left[t_{0}, T\right] \times\left(\mathcal{X}^{1}\right)^{r+1} \rightarrow \mathcal{Y}$ be valid for all $z_{0}, z_{1}, \ldots, z_{r} \in \mathcal{X}$, almost all $t \in\left(t_{0}, T\right)$. Let $Q N_{1}$ be uniformly Lipschitz continuous in $\bar{v}=\left(v_{0}, v_{1}, \ldots, v_{r}\right) \in\left(\mathcal{X}^{1}\right)^{r+1}$, for some $\bar{v} \in\left(\mathcal{X}^{1}\right)^{r+1}$, $Q N_{1}\left(\cdot, v_{0}, \ldots, v_{r}\right) \in L_{q}\left(t_{0}, T ; \mathcal{Y}\right),(I-Q) N_{1} \in C^{r}\left(\left[t_{0}, T\right] \times\left(\mathcal{X}^{1}\right)^{r+1} ; \mathcal{Y}\right),(I-Q) f \in W_{q}^{r}\left(t_{0}, T ; \mathcal{Y}\right)$, $Q f \in L_{q}\left(t_{0}, T ; \mathcal{Y}\right)$. Then for any $x_{0}, x_{1}, \ldots, x_{m-1} \in \mathcal{X}^{1}$ the problem $(3.1)-(3.2)$ has a unique strong solution on the interval $\left(t_{0}, T\right)$.

P r o o f. Multiply (3.1) from the left by the operators $L_{1}^{-1} Q$ or $M_{0}^{-1}(I-Q)$ and obtain the problem

$$
\begin{gathered}
D_{t}^{\alpha} v(t)=S_{1} v(t)+L_{1}^{-1} Q N_{1}\left(t, v(t), v^{(1)}(t), \ldots, v^{(r)}(t)\right)+L_{1}^{-1} Q f(t), \\
v^{(k)}\left(t_{0}\right)=P x_{k}, k=0,1, \ldots, m-1, \\
0=w(t)+M_{0}^{-1}(I-Q) N_{1}\left(t, v(t), v^{(1)}(t), \ldots, v^{(r)}(t)\right)+M_{0}^{-1}(I-Q) f(t)
\end{gathered}
$$


for the pair of functions $v(t) \equiv P x(t), w(t) \equiv(I-P) x(t)$. Here the notations $S_{1}=L_{1}^{-1} M_{1}$, $G=M_{0}^{-1} L_{0}$ are used.

By Theorem 2 the problem (3.4) has a unique strong solution, since the operator $S_{1}$ is bounded by Theorem 4 . Knowing $v$, obtain

$$
w(t)=-M_{0}^{-1}(I-Q) N_{1}\left(t, v(t), v^{(1)}(t), \ldots, v^{(r)}(t)\right)-M_{0}^{-1}(I-Q) f(t)
$$

from equation (3.5). Here $w \in W_{q}^{r}\left(t_{0}, T ; \mathcal{X}\right) \cap L_{q}\left(0, T ; D_{M}\right), L w \equiv 0$. Thus, there exists a unique strong solution $x=v+w$ of the problem (3.1)-(3.2).

A function $x \in C^{m-1}\left(\left[t_{0}, T\right] ; \mathcal{X}\right) \cap L_{q}\left(t_{0}, T ; D_{M}\right), q \in(1, \infty)$, is a strong solution of equation

$$
L D_{t}^{\alpha} x(t)=M x(t)+N\left(t, x(t), x^{(1)}(t), \ldots, x^{(r)}(t)\right)+f(t)
$$

on the interval $\left(t_{0}, T\right)$ if

$$
g_{m-\alpha} *\left(x-\sum_{k=0}^{m-1} x^{(k)}\left(t_{0}\right) g_{k+1}\right) \in W_{q}^{m}\left(t_{0}, T ; \mathcal{X}\right),
$$

and almost everywhere on $\left(t_{0}, T\right)$ the equality (3.6) is valid.

Theorem 6. Let $\alpha>1, q>(\alpha+1-m)^{-1}, r=0$, operator $M$ be $(L, 0)$-bounded, suppose that $N:\left[t_{0}, T\right] \times \mathcal{X} \rightarrow \mathcal{Y}$ for all $z \in \mathcal{X}, t \in\left[t_{0}, T\right]$ satisfies the equality $N(t, z)=N_{1}(t, P z)$ for some mapping $N_{1} \in C^{1}\left(\left[t_{0}, T\right] \times \mathcal{X}^{1} ; \mathcal{Y}\right),(I-Q) N_{1} \in C^{m}\left(\left[t_{0}, T\right] \times \mathcal{X}^{1} ; \mathcal{Y}\right), Q N_{1}$ is uniformly Lipschitz continuous in $v \in \mathcal{X}^{1}, f \in W_{q}^{1}\left(t_{0}, T ; \mathcal{Y}\right), q>(\alpha+1-m)^{-1},(I-Q) f \in C^{m}\left(\left[t_{0}, T\right] ; \mathcal{Y}\right)$, $x_{0}, x_{1}, \ldots, x_{m-1} \in \mathcal{X}^{1}$, the equality $Q N_{1}\left(t_{0}, P x_{0}\right)+Q f\left(t_{0}\right)=0$ is valid. Then there exists a unique strong solution of the problem (3.2), (3.6).

$\mathrm{P}$ r o o f. Arguing as in the proof of Theorem 5, obtain the unique solution $x=v+w$, where $v$ is an unique solution of the Cauchy problem for the equation $D_{t}^{\alpha} v(t)=S_{1} v(t)+L_{1}^{-1} Q N_{1}(t, v(t))+$ $L_{1}^{-1} Q f(t)$ and the function $w(t)=-M_{0}^{-1}(I-Q) N_{1}(t, v(t))-M_{0}^{-1}(I-Q) f(t)$. By Theorem 3 we have $v \in C^{m}\left(\left[t_{0}, T\right] ; \mathcal{X}\right)$, therefore $w \in C^{m}\left(\left[t_{0}, T\right] ; \mathcal{X}\right)$ and there exists $D_{t}^{\alpha} x \in L_{q}\left(t_{0}, T ; \mathcal{X}\right)$.

The proof of the next statement for the equation of an order $\alpha>2$, with $r \in\{1,2, \ldots, m-2\}$ is similar to the previous one.

Theorem 7. Let $\alpha>2, q>(\alpha+1-m)^{-1}, r \in\{1,2, \ldots, m-2\}$, operator $M$ be $(L, 0)$-bounded, suppose that $N:\left[t_{0}, T\right] \times \mathcal{X}^{r+1} \rightarrow \mathcal{Y}$ for all $z_{0}, z_{1}, \ldots z_{r} \in \mathcal{X}, t \in\left[t_{0}, T\right]$ satisfies condition (3.3) with some $N_{1} \in C^{r+1}\left(\left[t_{0}, T\right] \times\left(\mathcal{X}^{1}\right)^{r+1} ; \mathcal{Y}\right)$; a mapping $Q N_{1}$ is uniformly Lipschitz continuous in $\bar{v} \in \mathcal{X}^{r+1},(I-Q) N_{1} \in C^{m}\left(\left[t_{0}, T\right] \times\left(\mathcal{X}^{1}\right)^{r+1} ; \mathcal{Y}\right), f \in W_{q}^{r+1}\left(t_{0}, T ; \mathcal{Y}\right),(I-Q) f \in C^{m}\left(\left[t_{0}, T\right] ; \mathcal{Y}\right)$, $x_{0}, \ldots, x_{m-1} \in \mathcal{X}^{1}$; when $k=0,1, \ldots, m-1$ for the solution of problem

$$
\begin{gathered}
D_{t}^{\alpha} v(t)=S_{1} v(t)+L_{1}^{-1} Q N_{1}\left(t, v(t), v^{(1)}(t), \ldots, v^{(r)}(t)\right)+L_{1}^{-1} Q f(t), \\
v^{(l)}\left(t_{0}\right)=P x_{l}, l=0,1, \ldots, m-1,
\end{gathered}
$$

conditions

$$
\left.D_{t}^{k}\right|_{t=t_{0}} Q\left(N_{1}\left(t, v(t), v^{(1)}(t), \ldots, v^{(r)}(t)\right)+f(t)\right)=0, \quad k=0,1, \ldots, r,
$$

hold. Then problem (3.2), (3.6) has a unique strong solution on the interval $\left(t_{0}, T\right)$.

P r o of. The proof is similar to the previous one. Here we have $v \in C^{m+r}\left(\left[t_{0}, T\right] ; \mathcal{X}\right)$ by Theorem 3. 


\section{Optimal control problem}

Now let $\mathcal{X}, \mathcal{Y}, \mathcal{U}$ be Banach spaces, $L \in \mathcal{L}(\mathcal{X} ; \mathcal{Y})$, $\operatorname{ker} L \neq\{0\}, B \in \mathcal{L}(\mathcal{U} ; \mathcal{Y}), M \in \mathcal{C l}(\mathcal{X} ; \mathcal{Y})$ is $(L, p)$-bounded operator, $N:\left[t_{0}, T\right] \times \mathcal{X} \rightarrow \mathcal{Y}$. Consider the control problem

$$
\begin{gathered}
L D_{t}^{\alpha} x(t)=M x(t)+N(t, x(t))+B u(t), \\
(P x)^{(k)}\left(t_{0}\right)=x_{k}, k=0,1, \ldots, m-1, \\
u \in \mathcal{U}_{\partial}, \\
J(x, u) \rightarrow \inf ,
\end{gathered}
$$

where $\mathcal{U}_{\partial}$ is a set of admissible controls, the cost functional $J$ will be described below.

Taking into account the form of equation (4.1), we will seek its strong solutions in the linear space

$$
\mathcal{Z}_{\alpha, q}=\left\{x \in L_{q}\left(t_{0}, T ; D_{M}\right) \cap C^{m-1}\left(\left[t_{0}, T\right] ; \mathcal{X}\right): g_{m-\alpha} *\left(x-\sum_{k=0}^{m-1} x^{(k)}\left(t_{0}\right) g_{k+1}\right) \in W_{q}^{m}\left(t_{0}, T ; \mathcal{X}\right)\right\} .
$$

Lemma 3. For $q \in(\max \{1,1 / \alpha\}, \infty) \mathcal{Z}_{\alpha, q}$ is a Banach space with the norm

$$
\|x\|_{\mathcal{Z}}=\|x\|_{L_{q}\left(t_{0}, T ; D_{M}\right)}+\|x\|_{C^{m-1}\left(\left[t_{0}, T\right] ; \mathcal{X}\right)}+\left\|D_{t}^{\alpha} x\right\|_{L_{q}\left(t_{0}, T ; \mathcal{X}\right)} .
$$

P r o o f. Prove the closedness of the operator $D_{t}^{\alpha}: L_{q}\left(t_{0}, T ; D_{M}\right) \cap C^{m-1}\left(\left[t_{0}, T\right] ; \mathcal{Z}\right) \rightarrow$ $L_{q}\left(t_{0}, T ; \mathcal{Z}\right)$ with the domain $\mathcal{Z}_{\alpha, q}$. By definition of the Caputo fractional derivative $D_{t}^{\alpha}={ }^{R L} D_{t}^{\alpha} S_{m}$, where ${ }^{R L} D_{t}^{\alpha}$ is the Riemann-Liouville fractional derivative [1], we have

$$
S_{m} z \equiv z-\sum_{k=0}^{m-1} z^{(k)}\left(t_{0}\right) g_{k+1} .
$$

It is evident that the operator $S_{m}$ acts continuously from $\mathcal{Z}_{\alpha, q}$ with the norm of $C^{m-1}\left(\left[t_{0}, T\right] ; \mathcal{Z}\right)$ into the space

$$
\mathcal{R}_{\alpha, q, 0} \equiv\left\{z \in L_{q}\left(t_{0}, T ; \mathcal{Z}\right): g_{m-\alpha} * z \in W_{q, 0}^{m}\left(t_{0}, T ; \mathcal{Z}\right)\right\},
$$

endowed with the norm of $L_{q}\left(t_{0}, T ; \mathcal{Z}\right)$. And the operator ${ }^{R L} D_{t}^{\alpha}: \mathcal{R}_{\alpha, q, 0} \rightarrow L_{q}\left(t_{0}, T ; \mathcal{Z}\right)$ is closed by Lemma 1.8 (a) [1, p. 15].

Introduce the continuous operator $\gamma_{0}: C\left(\left[t_{0}, T\right] ; \mathcal{X}\right) \rightarrow \mathcal{X}, \gamma_{0} x=x\left(t_{0}\right)$.

The set of pairs $(x, u)$ will be called as admissible pairs set $\mathcal{W}$ of the problem (4.1)-(4.4) if $u \in \mathcal{U}_{\partial}, x \in \mathcal{Z}_{\alpha, q}$ is a strong solution of (4.1), (4.2), $J(x, u)<\infty$. Problem (4.1)-(4.4) is the problem of finding pairs $(\hat{x}, \hat{u}) \in \mathcal{W}$, which minimize the cost functional, i. e. $J(\hat{x}, \hat{u})=\inf _{(x, u) \in \mathcal{W}} J(x, u)$.

Theorem 8. Let $\alpha>1, q>(\alpha+1-m)^{-1}$, an operator $M$ be $(L, 0)$-bounded, $N:\left(t_{0}, T\right) \times$ $\mathcal{X} \rightarrow \mathcal{Y}$, for all $z \in \mathcal{X}, t \in\left(t_{0}, T\right) N(t, z)=N_{1}(t, P z)$ for some $N_{1} \in C^{1}\left(\left[t_{0}, T\right] \times \mathcal{X}^{1} ; \mathcal{Y}\right), Q N_{1}$ be uniformly Lipschitz continuous in $x \in \mathcal{X}^{1},(I-Q) N_{1} \in C^{m}\left(\left[t_{0}, T\right] \times \mathcal{X}^{1} ; \mathcal{Y}\right)$. Suppose that $\mathcal{U}_{\partial}$ is a non-empty closed convex subset of $L_{q}\left(t_{0}, T ; \mathcal{U}\right)$, there exists $u_{0} \in \mathcal{U}_{\partial} \cap W_{q}^{1}\left(t_{0}, T ; \mathcal{U}\right)$ such that $(I-Q) B u_{0} \in C^{m}\left(\left[t_{0}, T\right] ; \mathcal{U}\right), Q B u_{0}\left(t_{0}\right)=-Q N_{1}\left(t_{0}, P x_{0}\right) ; \mathcal{Z}_{\alpha, q}$ is continuously embedded in Banach space $\mathfrak{Y}, \mathfrak{Y}$ is continuously embedded in $L_{q}\left(t_{0}, T ; \mathcal{X}\right)$, cost functional $J$ is convex, lower semicontinuous, and bounded from below on $\mathfrak{Y} \times L_{q}\left(t_{0}, T ; \mathcal{U}\right)$, and $J$ is coercive on $\mathcal{Z}_{\alpha, q} \times L_{q}\left(t_{0}, T ; \mathcal{U}\right)$, $x_{k} \in \mathcal{X}^{1}, k=0,1, \ldots, m-1$. Then there exists a solution $(\hat{x}, \hat{u}) \in \mathcal{Z}_{\alpha, q} \times \mathcal{U}_{\partial}$ of the problem (4.1)$(4.4)$. 
P r o o f. The operator $N$ and the function $f=B u_{0}$ satisfy the conditions of Theorem 6 . Hence, Theorem 6 implies the existence of a strong solution of problem (4.1), (4.2) with $u=u_{0} \in \mathcal{U}_{\partial}$. So, the set of admissible pairs $\mathcal{W}$ is nonempty.

Further we will use Theorem 1.2.4 [14]. Put $\mathfrak{Y}_{1}=\mathcal{Z}_{\alpha, q}, \mathfrak{U}=L_{q}\left(t_{0}, T ; \mathcal{U}\right), \mathfrak{V}=L_{q}\left(t_{0}, T ; \mathcal{Y}\right) \times$ $\mathcal{X}^{m}, \mathfrak{F}(x(\cdot))=-\left(N(\cdot, x(\cdot)), x_{0}, x_{1}, \ldots, x_{m-1}\right), \mathfrak{L}(x, u)=\left(L D_{t}^{\alpha} x-M x-B u, \gamma_{0} P x, \ldots, \gamma_{0} P x^{(m-1)}\right)$. The continuity of the linear operator $\mathfrak{L}: \mathfrak{Y}_{1} \times \mathfrak{U} \rightarrow \mathfrak{V}$ follows from the inequalities

$$
\begin{aligned}
& \left\|\left(L D_{t}^{\alpha} x-M x-B u, \gamma_{0} P x, \gamma_{0} P x^{(1)}, \ldots, \gamma_{0} P x^{(m-1)}\right)\right\|_{L_{q}\left(t_{0}, T ; \mathcal{Y}\right) \times \mathcal{X}^{m}} \leq \\
& \leq C_{1}\left(\|x\|_{\mathcal{Z}_{\alpha, q}}+\|u\|_{L_{q}\left(t_{0}, T ; \mathcal{U}\right)}+\|x\|_{C^{m-1}\left(\left[t_{0}, T\right] ; \mathcal{X}\right)}\right) \leq C_{2}\|(x, u)\|_{\mathcal{Z}_{\alpha, q} \times \mathcal{U}}
\end{aligned}
$$

From the relation $\left\|x_{n}-x\right\|_{\mathcal{Z}_{\alpha, q}} \rightarrow 0$ for $n \rightarrow \infty$ it follows that

$$
\left\|N\left(\cdot, x_{n}(\cdot)\right)-N(\cdot, x(\cdot))\right\|_{L_{q}\left(t_{0}, T ; \mathcal{Y}\right)} \leq C_{1}\left\|x_{n}-x\right\|_{C\left(\left[t_{0}, T\right] ; \mathcal{X}\right)} \rightarrow 0,
$$

therefore the operator $\mathfrak{F}$ is continuous.

After choosing $\mathfrak{Y}_{-1}=L_{q}\left(t_{0}, T ; \mathcal{X}\right)$, check the remaining conditions of Theorem 1.2.4 [14]. From Rellich-Kondrashov theorem it follows that $\mathcal{Z}_{\alpha, q}$ enclosed to $W_{q}^{m-1}\left(t_{0}, T ; \mathcal{X}\right)$ and compactly enclosed to $L_{q}\left(t_{0}, T ; \mathcal{X}\right)$. For $v \in\left(L_{q}\left(t_{0}, T ; \mathcal{Y}\right)\right)^{*}$ the uniform Lipschitz continuity of the operator $N$ implies the inequality

$$
\mid v\left(N\left(t, x_{n}(t)\right)-N(t, x(\cdot)) \mid \leq C_{1}\|v\|_{\left(L_{q}\left(t_{0}, T ; \mathcal{Y}\right)\right)^{*}}\left\|x_{n}-x\right\|_{L_{q}\left(t_{0}, T ; \mathcal{X}\right)} .\right.
$$

It gives the continuous extension of the functional $f(\cdot)=v(\mathfrak{F}(\cdot))$ from $\mathcal{Z}_{\alpha, q}$ to $L_{q}\left(t_{0}, T ; \mathcal{X}\right)$.

In applications the condition of the uniform Lipschitz continuity of $N$ is too strong. But the nonemptyness of $\mathcal{W}$ is often evident. Consider the optimal control problem in such case.

A mapping $N \in C\left(\left[t_{0}, T\right] \times \mathcal{X} ; \mathcal{Y}\right)$ will be called locally Lipschitz continuous in $x \in \mathcal{X}$, uniformly with respect to $t \in\left[t_{0}, T\right]$, if for every $x \in \mathcal{X}$ there exists $\delta>0$ and $l>0$ such that for every $y \in \mathcal{Y}$ the inequality $\|y-x\|_{\mathcal{X}}<\delta$ implies that $\|N(t, y)-N(t, x)\|_{\mathcal{Y}} \leq l\|y-x\|_{\mathcal{X}}$ for all $t \in\left[t_{0}, T\right]$.

Theorem 9. Let $\alpha, q>1$, an operator $M$ be $(L, p)$-bounded, the mapping $N \in C\left(\left[t_{0}, T\right] \times \mathcal{X} ; \mathcal{Y}\right)$ be locally Lipschitz continuous in $z \in \mathcal{X}$, uniformly with respect to $t \in\left[t_{0}, T\right]$. Suppose that $x_{k} \in \mathcal{X}^{1}$, $k=0,1, \ldots, m-1, \mathcal{U}_{\partial}$ is a non-empty closed convex subset of $L_{q}\left(t_{0}, T ; \mathcal{U}\right)$, for some $u_{0} \in \mathcal{U}_{\partial}$ there exists a solution of the problem (4.1)-(4.2); $\mathcal{Z}_{\alpha, q}$ is continuously embedded in Banach space $\mathfrak{Y}, \mathfrak{Y}$ is continuously embedded in $L_{q}\left(t_{0}, T ; \mathcal{X}\right)$, cost functional $J$ is convex, lower semicontinuous, and bounded from below on $\mathfrak{Y} \times L_{q}\left(t_{0}, T ; \mathcal{U}\right)$, and $J$ is coercive on $\mathcal{Z}_{\alpha, q} \times L_{q}\left(t_{0}, T ; \mathcal{U}\right)$. Then there exists a solution $(\hat{x}, \hat{u}) \in \mathcal{Z}_{\alpha, q} \times \mathcal{U}_{\partial}$ of the problem (4.1)-(4.4).

P r o o f. The set $\mathcal{W}$ is non-empty by the conditions of the theorem. The conditions on the mapping $N$ are sufficient for repeating the previous proof.

\section{Optimal control for fractional Kelvin-Voigt fluid}

Consider a control problem

$$
\begin{gathered}
(1-\chi \Delta) D_{t}^{\alpha} v(s, t)=\nu \Delta v(s, t)-(v \cdot \nabla) v(s, t)-r(s, t)+u(s, t),(s, t) \in \Omega \times[0, T], \\
\nabla \cdot v(s, t)=0, \quad(s, t) \in \Omega \times[0, T], \\
v(s, t)=0, \quad(s, t) \in \partial \Omega \times[0, T],
\end{gathered}
$$




$$
\begin{gathered}
\frac{\partial^{k} v}{\partial t^{k}}(s, 0)=\psi_{k}(s), \quad k=0,1, \ldots, m-1, \quad s \in \Omega, \\
\|u\|_{L_{q}\left(0, T ; \mathbb{L}_{2}\right)} \leq R, \\
J(v, r, u)=\left\|v-v_{d}\right\|_{C^{m-1}\left([0, T] ; \mathbb{H}_{\sigma}^{2}\right)}+\left\|r-r_{d}\right\|_{C^{m-1}\left([0, T] ; \mathbb{H}_{\pi}\right)}+ \\
+\left\|D_{t}^{\alpha} v-D_{t}^{\alpha} v_{d}\right\|_{L_{q}\left(0, T ; \mathbb{H}_{\sigma}^{2}\right)}^{q}+\left\|D_{t}^{\alpha} r-D_{t}^{\alpha} r_{d}\right\|_{L_{q}\left(0, T ; \mathbb{H}_{\pi}\right)}^{q}+\left\|u-u_{d}\right\|_{L_{q}\left(0, T ; \mathbb{L}_{2}\right)}^{q} \rightarrow \inf .
\end{gathered}
$$

Here, $\Omega \subset \mathbb{R}^{3}$ is a domain with a smooth boundary $\partial \Omega, \chi, \nu \in \mathbb{R}, T>0$. The vector-functions $\psi_{k}=\left(\psi_{k 1}, \psi_{k 2}, \psi_{k 3}\right): \Omega \rightarrow \mathbb{R}^{3}, k=0,1, \ldots, m-1$, are set. Vector-functions $v=\left(v_{1}, v_{2}, v_{3}\right)$ of the velocity and $r=\left(r_{1}, r_{2}, r_{3}\right)=\left(p_{s_{1}}, p_{s_{2}}, p_{s_{3}}\right)$ of the pressure $p$ gradient are unknown. An external source $u=\left(u_{1}, u_{2}, u_{3}\right): \Omega \times[0, T] \rightarrow \mathbb{R}^{3}$ is a control function. The system models the dynamics of a fractional viscoelastic incompressible Kelvin — Voigt fluid [2].

To reduce the optimal control problem (5.1)-(5.6) to problem (4.1)-(4.4), denote the Lebesgue space $\mathbb{L}_{2}=\left(L_{2}(\Omega)\right)^{3}$, and the Sobolev spaces $\mathbb{H}^{1}=\left(W_{2}^{1}(\Omega)\right)^{3}, \mathbb{H}^{2}=\left(W_{2}^{2}(\Omega)\right)^{3}$ of vector-functions $w=\left(w_{1}, w_{2}, w_{3}\right)$, defined in $\Omega$. A closure of the lineal $\mathcal{L}=\left\{w \in\left(C_{0}^{\infty}(\Omega)\right)^{3}: \nabla \cdot w=0\right\}$ by the norm in $\mathbb{L}_{2}$ is denoted by $\mathbb{H}_{\sigma} ; \mathbb{H}_{\sigma}^{1}$ is its closure by the norm in $\mathbb{H}^{1}$. Also, we use $\mathbb{H}_{\sigma}^{2}=\mathbb{H}_{\sigma}^{1} \cap \mathbb{H}^{2}$. An orthogonal complement to $\mathbb{H}_{\sigma}$ in $\mathbb{L}_{2}$ is denoted by $\mathbb{H}_{\pi}$. The corresponding orthoprojectors are $\Sigma: \mathbb{L}_{2} \rightarrow \mathbb{H}_{\sigma}, \Pi=I-\Sigma: \mathbb{L}_{2} \rightarrow \mathbb{H}_{\pi}$.

Consider an operator $A=\Sigma \Delta$ in $\mathcal{L}$. The operator $A$, extended to a closed operator in $\mathbb{H}_{\sigma}$, with a domain $\mathbb{H}_{\sigma}^{2}$, is known (see [23]) to have a real, negative discrete spectrum of finite multiplicity, condensing at $-\infty$ only. Its eigenvalues are denoted by $\left\{\lambda_{k}\right\}$, numbered in non-increasing, counting their multiplicities. The orthonormal system of corresponding eigenfunctions $\left\{\varphi_{k}\right\}$ is known to form a basis in $\mathbb{H}_{\sigma}$.

Choose spaces and operators as

$$
\begin{gathered}
\mathcal{X}=\mathbb{H}_{\sigma}^{2} \times \mathbb{H}_{\pi}, \quad \mathcal{Y}=\mathbb{L}_{2}=\mathbb{H}_{\sigma} \times \mathbb{H}_{\pi}, \quad \mathcal{U}=\mathbb{L}_{2}, \\
L=\left(\begin{array}{cc}
I-\chi A & \mathbb{O} \\
-\chi \Pi \Delta & \mathbb{O}
\end{array}\right), \quad M=\left(\begin{array}{cc}
\nu A & \mathbb{O} \\
\nu \Pi \Delta & -I
\end{array}\right) \in \mathcal{L}(\mathcal{X} ; \mathcal{Y}) .
\end{gathered}
$$

Lemma 4. Let spaces $\mathcal{X}$ and $\mathcal{Y}$ be defined in (5.7), and operators $L$ and $M$ be defined in (5.8), $\nu, \chi \neq 0, \chi^{-1} \notin \sigma(A)$. Then $M$ is $(L, 0)$-bounded operator, and

$$
P=\left(\begin{array}{cc}
I & \mathbb{O} \\
\nu \Pi \Delta(I-\chi A)^{-1} & \mathbb{O}
\end{array}\right), \quad Q=\left(\begin{array}{cc}
I & \mathbb{O} \\
-\chi \Pi \Delta(I-\chi A)^{-1} & \mathbb{O}
\end{array}\right) .
$$

Denote

$$
\Psi(s, t)=\psi_{0}(s)+\psi_{1}(s) t+\cdots+\psi_{m-1}(s) \frac{t^{m-1}}{(m-1) !} .
$$

Theorem 10. Let $\nu, \chi \neq 0, \chi^{-1} \notin \sigma(A), \alpha, q>1, \psi_{k} \in \mathbb{H}_{\sigma}^{2}, k=0,1, \ldots, m-1$, the inequality

$$
\left\|(1-\chi \Delta) D_{t}^{\alpha} \Psi-\nu \Delta \Psi+(\Psi \cdot \nabla) \Psi\right\|_{L_{q}\left(0, T ; \mathbb{L}_{2}\right)} \leq R
$$

is true. Then there exists a solution of the problem (5.1)-(5.6).

P r o o f. From the form of the projector $P$ it follows that (5.4) are Showalter - Sidorov conditions. Besides, there exists a control

$$
u_{0}=(1-\chi \Delta) D_{t}^{\alpha} \Psi-\nu \Delta \Psi+(\Psi \cdot \nabla) \Psi \in \mathcal{U}_{\partial}=\left\{u \in L_{q}\left(0, T ; \mathbb{L}_{2}\right):\|u\|_{L_{q}\left(0, T ; \mathbb{L}_{2}\right)} \leq R\right\},
$$

such that $(\Psi, 0)(r=0)$ is a strong solution of the problem (5.1)-(5.4) with $u=u_{0}$, i. e. $\left(\Psi, 0, u_{0}\right) \in \mathcal{W}$. 
Define $N(v)=-(v \cdot \nabla) v$, hence, by Sobolev's embedding theorem

$$
\|N(v)\|_{\mathbb{L}_{2}}^{2} \leq C_{1}\|v\|_{\mathbb{W}_{4}^{1}}^{4} \leq C_{2}\|v\|_{\mathbb{H}^{2}}^{4}
$$

where $\mathbb{W}_{4}^{1}=\left(W_{4}^{1}(\Omega)\right)^{3}$. Besides, $N$ doesn't depend on $r$ and is locally Lipschitzian mapping.

Choose $\mathfrak{Y}=\left\{(v, r) \in C^{m-1}([0, T] ; \mathcal{X}):\left(D_{t}^{\alpha} v, D_{t}^{\alpha} r\right) \in L_{q}(0, T ; \mathcal{X})\right\}$ with the norm

$$
\|x\|_{\mathfrak{Y}}=\|x\|_{C^{m-1}\left(\left[t_{0}, T\right] ; \mathcal{X}\right)}+\left\|D_{t}^{\alpha} x\right\|_{L_{q}\left(t_{0}, T ; \mathcal{X}\right)}, \quad x=(v, r) .
$$

The completeness of $\mathfrak{Y}$ can be shown as in the proof of Lemma 4. The functional $J$ is coercive on $\mathcal{Z}_{\alpha, q}$ because of the estimate

$$
\|M x\|_{L_{q}\left(0, T ; \mathbb{L}_{2}\right)} \leq C_{1}\left\|D_{t}^{\alpha} v\right\|_{L_{q}\left(0, T ; \mathbb{H}_{\sigma}^{2}\right)}+\|u\|_{L_{q}\left(0, T ; \mathbb{L}_{2}\right)}+\max _{\|v\|_{\mathbb{H}_{\sigma}^{2}} \leq 1}\|N(v)\|_{\mathbb{L}_{2}}
$$

The required statement follows from Theorem 9.

\section{REFERENCES}

1. Bajlekova E.G. Fractional Evolution Equations in Banach Spaces // PhD thesis, Eindhoven University of Technology, University Press Facilities, 2001.

2. Mainardi F., Spada G. Creep, relaxation and viscosity properties for basic fractional models in rheology // The European Physics Journal, Special Topics, 2011. Vol. 193. P. 133-160.

3. Sviridyuk G.A., Fedorov V.E. Linear Sobolev Type Equations and Degenerate Semigroups of Operators. VSP, Utrecht, Boston, 2003.

4. Fedorov V.E., Davydov P.N. On nonlocal solutions of semilinear equations of the Sobolev type // Differential Equations. 2013. Vol. 49, no. 3. P. 338-347. DOI: 10.1134/S0012266113030087

5. Fedorov V.E., Gordievskikh D.M. Resolving operators of degenerate evolution equations with fractional derivative with respect to time // Russian Math., 2015. Vol. 59. P. 60-70. DOI:10.3103/S1066369X15010065

6. Fedorov V.E., Gordievskikh D.M., Plekhanova M.V. Equations in Banach spaces with a degenerate operator under a fractional derivative // Differential Equations, 2015. Vol. 51. P. 1360-1368.

7. Fedorov V.E., Debbouche A. A class of degenerate fractional evolution systems in Banach spaces // Differential Equations, 2013. Vol. 49, no. 12. P. 1569-1576. DOI: 10.1134/S0012266113120112.

8. Gordievskikh D.M., Fedorov V.E. Solutions for initial boundary value problems for some degenerate equations systems of fractional order with respect to the time // The Bulletin of Irkutsk State University. Series Mathematics, 2015. Vol. 12. P. 12-22.

9. Plekhanova M.V. Quasilinear equations that are not solved the higher-order time derivative // Siberian Mathematical Journal, 2015. Vol. 56. P. 725-735.

10. Plekhanova M.V. Nonlinear equations with degenerate operator at fractional Caputo derivative // Mathematical Methods in the Applied Sciences. 2016. In press. DOI: 10.1002/mma.3830

11. Kostic M. Abstract time-fractional equations: Existence and growth of solutions // Fractional Calculus and Applied Analysis. 2011. Vol. 14, no. 2. P. 301-316.

12. Glushak A.V. Correctness of Cauchy-type problems for abstract differential equations with fractional derivatives // Russian Mathematics. 2009. Vol. 53, no. 9. P. 10-19.

13. Vorob'eva S.A., Glushak A.V. An abstract EulerPoissonDarboux equation containing powers of an unbounded operator // Differential Equations. 2001. Vol. 37, no. 5. P. 743-746.

14. Fursikov, A.V. Optimalnoe upravlenie raspredelennymi sistemami. Teoriya i prilozheniya. Optimal Control of Distributed Systems. Theory and Applications, Novosibirsk, 1999. [In Russian]

15. Fedorov V.E., Plekhanova M.V. Optimal control of Sobolev type linear equations // Differential equations, 2004. Vol. 40. P. 1548-1556.

16. Fedorov V.E., Plekhanova M.V. The problem of start control for a class of semilinear distributed systems of Sobolev type // Proceeding of the Steklov institute if mathematics. 2011. Vol. 275. P. 40-48. 
17. Plekhanova M.V. Distributed control problems for a class of degenerate semilinear evolution equations // J. of Computational and Applied Mathematics, 2017. Vol. 312. P. 39-46. http://dx.doi.org/10.1016/j.cam.2015.09.034

18. Kochubei N. Fractional-parabolic systems // Potential Anal. 2012. Vol. 37. P. 1-30.

19. Kamocki R. On the existence of optimal solutions to fractional optimal control problems // Applied Mathematics and Computation. 2014. Vol. 235. P. 94-104.

20. Kerboua M., Debbouche A., Baleanu D. Approximate controllability of Sobolev type fractional stochastic nonlocal nonlinear differential equations in Hilbert spaces // Electronic J. of Qualitative Theory of Differential Equations, 2014. Vol. 58. P. 1-16.

21. Showalter R.E. Nonlinear degenerate evolution equations and partial differential equations of mixed type // SIAM J. Math. Anal., 1975. Vol. 6. P. 25-42. DOI: 10.1137/0506004

22. Sidorov N.A. A class of degenerate differential equations with convergence // Math. Notes., 1984. Vol. 35. P. 300-305.

23. Ladyzhenskaya O.A. The Mathematical Theory of Viscous Incompressible Flow. Gordon and Breach, Science Publishers, New York, London, Paris, 1969. 